\title{
Composite films of poly-(ester-sulphonated) and poly-(3-methylthiophene) for ion-exchange voltammetry in acetonitrile solutions
}

\author{
Paolo Scopece ${ }^{1}$, Ligia M. Moretto, Stefano Polizzi, Paolo Ugo* \\ Department of Physical Chemistry, University of Venice, Calle Larga Santa Marta 2137, \\ 30123 Venice, Italy
}

Received 26 November 2004; received in revised form 19 March 2005; accepted 30 March 2005

Available online 1 September 2005

\begin{abstract}
This paper describes the preparation and characterisation of a polymeric electrode coating based on a composite of the poly-(estersulphonated) Eastman AQ55 ${ }^{\circledR}$ (AQ55) and poly-(3-methylthiophene) (PMeT), which is used for the controlled uptake and partial release of electroactive cations in acetonitrile solutions. The film is prepared by electrochemical oxidation in acetonitrile of 3-methylthiophene on glassy carbon disks or Pt-quartz crystal electrodes pre-coated with a thin film of AQ55.

The electropolymerisation process is controlled so that the overall number of positive charges of oxidised PMeT is equal to the number of negative charges of the sulphonate groups of AQ55. Cyclic voltammetry and quartz crystal microbalance measurements indicate that the AQ55/PMeT mixed film is stable in acetonitrile and that its cation-exchange properties depend on the applied potential. When the PMeT moieties are reduced, the film incorporate cations; following electrochemical oxidation of the coating causes a release of the incorporated cations which, however, is only partial.

Scanning electron microscopy (SEM) examination of cross sections of the composite polymer layer indicate that it is really a bi-layer, made by an inner compact layer of AQ55 on which a thicker and porous PMeT layer is grown. The outer PMeT layer acts as a barrier whose ionic charges can be changed electrochemically from positive (oxidation) to neutral (reduction). These ionic charges hinder or allow, respectively, the permeation of redox cations which tend to interact with the negatively charged sulphonic sites of the AQ55 layer. Direct self-neutralization of part of the positive charges of oxidized PMeT by the AQ55 sulphonic groups allows the release of part of the redox cations incorporated previously in the mixed film when PMeT is in the reduced state. By operating in acetonitrile solutions without added electrolyte it is possible to increase the fraction of redox cations which are released in consequence of the oxidation of PMeT; this suggests a slower and only partial oxidation of PMeT under such experimental conditions.
\end{abstract}

(C) 2005 Elsevier Ltd. All rights reserved.

Keywords: Ion-exchange; Voltammetry; Poly-(3-methylthiophene); Poly-(ester-sulphonated); Acetonitrile

\section{Introduction}

The possibility to introduce electrochemical control in the uptake and release of electroactive cations in polymercoated electrodes is a very attractive property which has been

\footnotetext{
* Corresponding author.

E-mail address: ugo@unive.it (P. Ugo).

${ }^{1}$ Present address: CIVEN Coordinamento Interuniversitario Veneto Nanotecnologie, Via delle Industrie 17/a, Marghera, Venice, Italy.
}

examined in numerous studies [1]. To this aim, two main approaches were followed: one based on the use of electroactive films with electrochemically controllable ion-exchange properties such as redox polymers [2-5] or conducting polymers [6,7], the other taking advantage of the special properties of composite films in which the (switchable) anionexchange properties of oxidized conducting polymers were compensated and combined with the cation-exchange properties of polyelectrolytes [8-17]. Very recently, these studies brought to the proposal of using electrochemically controlled 
films for performing and controlling electrochemically the solid phase micro extraction (SPME) of anions or cations $[9,15,18,19]$.

However, all these studies were devoted in examining the electrochemical switching of the uptake-release of trace analytes in water solutions, but few, if none, studies in this direction were devoted to applications in non-aqueous media. This appears quite a relevant lack in view of the fact that many trace environmental pollutants are characterized by poor water solubility and water is not the only relevant matrix where these pollutants can be present. Moreover, the preparation of ion-exchange coatings whose properties can be controlled electrochemically in organic solvents appears relevant not only for SPME aims but also for their possible application as electrochemically modulated stationary phases in liquid chromatography [20-23], where organic solvents, in general, and acetonitrile, in particular, are very often used as mobile phases.

Until now, developments in this direction were limited by the impossibility to use the most applied ion-exchange film coating, namely Nafion ${ }^{\circledR}$, in non-aqueous solutions. A valid alternative to Nafion is offered by the poly-(estersulphonated) Eastman $\mathrm{AQ} 55^{\circledR}$ (AQ55), which is stable and presents good cation-exchange properties in acetonitrile solutions [24-27].

In order to develop a layer suitable as electrode coating for electrochemically controlled ion-exchange in non-aqueous solvent, in the present work we report on the preparation and characterization of an electrode coating obtained by electropolymerisation of 3-methylthiophene (MeT) on AQ55, with the aim of combining the good cation exchange properties of the latter with the redox switchability of the former [9,28-32].

Quantitative data on the ion-exchange properties and electrochemical switching of the film in acetonitrile solutions are obtained studying the uptake and release of the cationic complex tris $\left(2,2^{\prime}\right.$ bipyridil) osmium(II) $\left.(\mathrm{Os} \text { (bpy })_{3}{ }^{2+}\right)$, used as reversible redox probe of well known behaviour [26]. By this way, it was possible to gain fundamental information on the properties of the mixed film in acetonitrile solutions as a necessary basis for future possible application for the uptake-release of redox analytes of environmental interest.

\section{Experimental}

\subsection{Chemicals}

All chemicals used were of analytical grade quality. Reagent grade acetonitrile was purified further by distillation from phosphorus pentoxide and stored under nitrogen atmosphere. The supporting electrolytes tetrabutylammonium tetrafluoroborate (TBATFB) and tetrabutylammonium hexafluorophosphate (TBAHFP) (both from Fluka, puriss.) were dried overnight in a vacuum oven at $50^{\circ} \mathrm{C}$ before use. The Eastman AQ55 $5^{\circledR}$ polymer (supplied as irregular shaped pellets) was kindly provided as a gift by Eastman Italia. Chloride salt of tris $\left(2,2^{\prime}\right.$ bipyridil) osmium(II) was prepared according to the procedures described in the literature [33]. Chloride salts of the synthesized complexes were converted into hexafluorophosphate salts by reaction with a saturated aqueous solution of $\mathrm{KPF}_{6}$.

\subsection{Electrochemical apparatus and procedures}

Cyclic voltammetric measurements were performed with a CHI 620A instrument controlled by its own software. EQCM determinations were carried out using an EG\&G-PAR 273 potentiostat/galvanostat interconnected with an EG\&GSeiko quartz crystal analyser, using $9 \mathrm{MHz}$ AT-cut quartz crystals coated with platinum ( $\mathrm{Pt}$ area $0.2 \mathrm{~cm}^{2}$ ). The crystal sensitivity, measured as in ref. [34] was $0.192 \mathrm{~Hz} \mathrm{~cm}^{2} \mathrm{ng}^{-1}$. For CV and EQCM measurements, a three electrodes cell was employed equipped with a glassy carbon disk (GC, $5 \mathrm{~mm}$ diameter) or a Pt-coated quartz crystal working electrode (both bare or polymer coated), a Pt wire counter electrode, an aqueous $\mathrm{Ag} / \mathrm{AgCl}(\mathrm{KCl}$ sat) reference electrode separated from the sample solution by a salt bridge filled with the acetonitrile electrolyte solution. All potentials reported in this paper are referred to this reference electrode. For the galvanostatic and potentiostatic electropolymerization, an Amel 552 potentiostat/galvanostat was employed using a two electrodes cell (working and Pt spiral counter electrode). The sample solution was purged first with purified nitrogen for $15 \mathrm{~min}$ and was held under a nitrogen atmosphere throughout. All experiments were conducted at room temperature $\left(22 \pm 2{ }^{\circ} \mathrm{C}\right)$.

\subsection{Preparation of film modified electrodes}

The AQ55 aqueous dispersion (approximately 12\%, w/v) was prepared by treating the proper amount of the finely grinded AQ55 resin with warm water $\left(45^{\circ} \mathrm{C}\right)$, under vigorous stirring until a homogeneous and transparent phase was obtained [24]. The exact concentration of the polymer in the dispersion was determined by drying and weighing a known volume of this dispersion.

GC electrodes (mirror polished with graded alumina powder) were modified with AQ55 by deposition of a microvolume ( $4 \mu 1$, with a microsyringe) of an acetone/water solution obtained by $1: 1(\mathrm{v} / \mathrm{v})$ dilution with acetone of the AQ55 $12 \%$ aqueous dispersion, letting the solvent to evaporate at room temperature (approximately $30 \mathrm{~min}$ ). The number of ion-exchange sites, estimated on the basis of the equivalent weight of 1700 [24], is $7.2 \times 10^{-7} \mathrm{~cm}^{-2}$.

For EQCM measurements, in order to operate in the field of applicability of the Sauerbray equation, it was required to obtain thinner films, with thickness $\leq 0.6 \mu \mathrm{m}$ [35]. For this reason a spin-coating procedure was employed. Namely, $20 \mu \mathrm{L}$ of above described acetone/water solution of AQ55 were deposited on the Pt-quartz crystal electrode, attached at a spinner rotating at $3800 \mathrm{rpm}$; the film was dried, under 
spinning conditions, for 10 min under a warm air flux by an hair-drier. The mass of AQ55 deposited on the Pt surface was calculated by frequency measurements, before and after the spin-coating procedure; the average deposited mass was $10 \mu \mathrm{g}$. From the known density of AQ55, which is $1.34 \mathrm{~g} / \mathrm{cm}^{3}$ [36], the thickness of the AQ55 film on the Pt electrode was estimated to be about $0.45 \mu \mathrm{m}$. Finally, from the average equivalent weight of AQ55, the number of sulphonic ion-exchange sites in the deposited coating was estimated to be $3.6 \times 10^{-8} \mathrm{~cm}^{-2}$. Before the use in the sample solution, the coated electrodes were equilibrated with the electrolyte solution by dipping in acetonitrile/supporting electrolyte, for at least $30 \mathrm{~min}$.

Electropolymerization of MeT on bare Pt-quartz crystal electrodes was carried out by cyclic voltammetry in $0.1 \mathrm{M}$ TBATFB, $20 \mathrm{mM}$ MeT, acetonitrile solutions.

As far as the AQ55/poly-(3-methylthiophene) (PMeT) composite film preparation is concerned, attempts to perform the electropolymerization of $\mathrm{MeT}$ in acetonitrile solution containing AQ55 as polymeric electrolyte were unsuccessful because of the insolubility of the poly-(ester-sulphonated) ionomer in this medium. Tests performed in acetonitrile/water solution, in the presence of dissolved AQ55, were also unsuccessful since no deposit was obtained. Finally, MeT polymerization was possible using electrodes precoated with AQ55. Electropolymerization of MeT on GC-AQ55 coated electrodes was performed both potentiostatically and galvanostatically; in the latter case a current of $0.3 \mathrm{~mA}$ for $60 \mathrm{~s}$ in a $50 \mathrm{mM} \mathrm{MeT,} \mathrm{0.1} \mathrm{M} \mathrm{TBATFB} \mathrm{acetonitrile} \mathrm{solution} \mathrm{was} \mathrm{used.}$ Under such conditions, the number of the positive charges introduced by PMeT deposition was calculated by the Faraday's law.

The electropolymerization of MeT on Pt-quartz crystal electrodes spin-coated with AQ55 was performed potentiostatically, at a constant applied potential of $1.8 \mathrm{~V}$ (versus $\mathrm{Ag} \mid \mathrm{AgCl}$ ) in $20 \mathrm{mM}$ MeT, 0.1 M TBATFB acetonitrile solution.

\subsection{Scanning electron microscopy (SEM)}

Scanning electron microscopy images were taken with a Jeol JSM 5600 LV equipped with an Oxford Instrument 6587 EDS microanalysis detector. Cross sections were obtained by fracturing the sample at room temperature or after immersion in liquid nitrogen. Samples were coated with an Au thin film in order to avoid charging effects.

\section{Results and discussion}

\subsection{Electropolymerization of 3-methylthiophene}

The electrochemical polymerization of MeT has been studied in detail [28-32], however, some points of interest for the preparation of the mixed film are revisited here. Fig. 1 shows the growth of the PMeT film on the Pt-quartz elec-

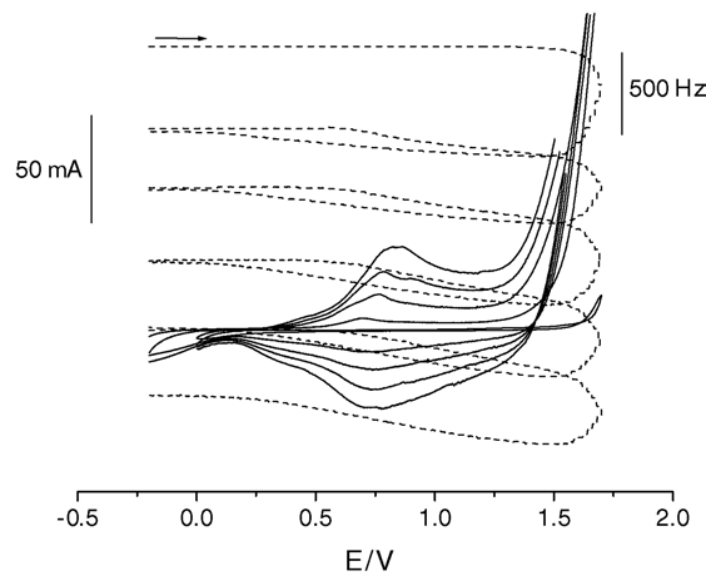

Fig. 1. Continuous scan cyclic voltammograms (full line, left hand $Y$-axis) of $20 \mathrm{mM} \mathrm{MeT}$ in $0.1 \mathrm{M}$ TBATFB acetonitrile solution at Pt-coated quartz crystal electrode and concomitant resonator frequency variations (dotted line, right hand $Y$-axis). Initial and final potential $-0.2 \mathrm{~V}$, vertex potential $1.7 \mathrm{~V}$, scan rate $20 \mathrm{mV} / \mathrm{s}$.

trode, studied by cyclic voltammetry and concomitant EQCM measurements. In agreement with the literature [17], the oxidation branch of the first scan is flat until an uprise of the current is observed at $E>1.5 \mathrm{~V}$. The monomer is oxidized indeed at these potential values, producing the corresponding polymer through radical type reactions [29].

After reversing the direction of the sweep, a reduction wave is observed at about $+0.75 \mathrm{~V}$, followed by a smaller one at about $0.4 \mathrm{~V}$. Such processes are related to the electroactivity of the deposited polymer (see below) according to reaction (1):

$$
\begin{aligned}
& {\left[\mathrm{PMeT}^{\delta n+}\left(\mathrm{BF}_{4}{ }^{-}\right)_{\delta n}\right]+\delta n \mathrm{TBA}^{+}+\delta n \mathrm{e}^{-}} \\
& \leftrightarrows \mathrm{PMeT}+\delta n\left(\mathrm{TBA}^{+} \mathrm{BF}_{4}{ }^{-}\right)
\end{aligned}
$$

The following oxidation sweeps revealed the two following processes: at first the oxidation of the deposited polymer with a peak potential of $0.7 \mathrm{~V}$ and, at more positive potentials, the further growth of the polymer film. The occurrence of the latter process is confirmed by the progressive increase in the voltammetric peak currents for successive scans. EQCM plots, reported in the same Fig. 1, show relevant frequency changes. Each EQCM cycle (apart the first) is characterized by two different kinds of frequency change:

1) the doping-undoping process which parallels the oxidation of the previously deposited polymer, in the $0.2 \mathrm{~V}<E<1.5 \mathrm{~V}$ region;

2) the further growing of the polymer film, which occurs at potentials $>1.5 \mathrm{~V}$.

Apart from the first cycle, the average frequency change associated to each oxidation-reduction cycle is about $470 \mathrm{~Hz}$. The application of the Sauerbrey equation [35] gives a mass change of $2.4 \mu \mathrm{g} / \mathrm{cm}^{2}$ per cycle.

In order to focus only on the uptake and release of ions from the supporting electrolyte solution, some scans were 

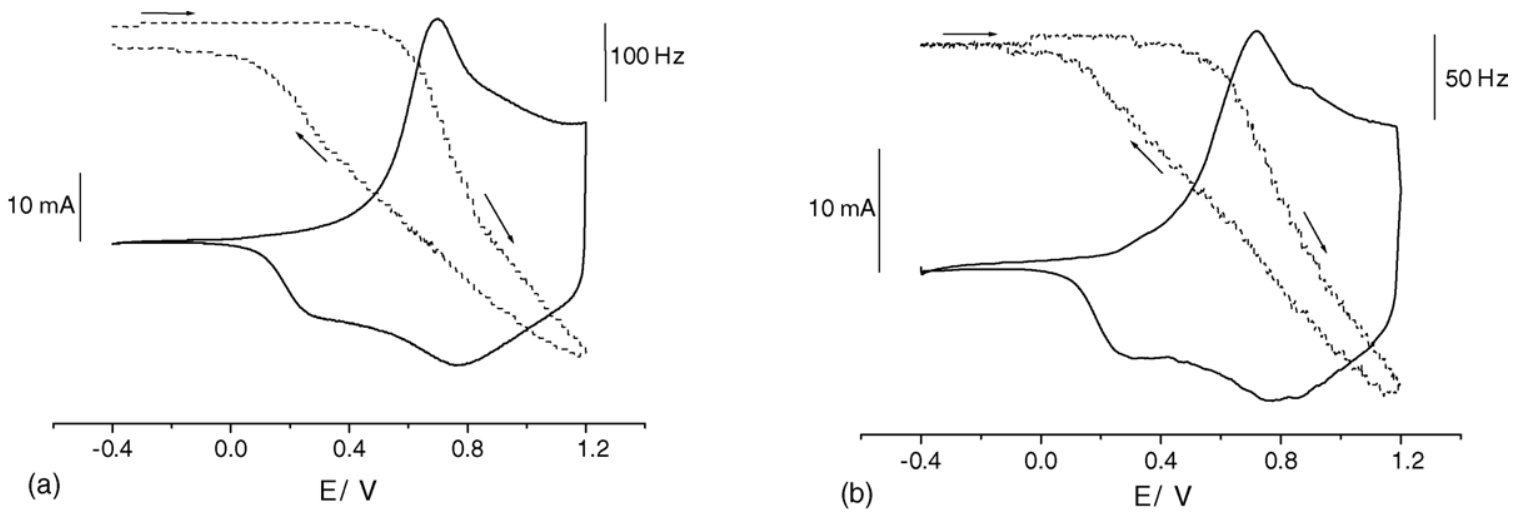

Fig. 2. (a) Cyclic voltammogram (full line, left hand $Y$-axis) of $2.64 \mu \mathrm{g}\left(13.5 \mu \mathrm{g} / \mathrm{cm}^{2}\right)$ PMeT modified electrode in $0.1 \mathrm{M}$ TBATFB acetonitrile solution and concomitant resonator frequency variation (dotted line, right hand $Y$-axis). Scan rate $20 \mathrm{mV} / \mathrm{s}$. (b) Cyclic voltammogram (full line, left hand $Y$-axis) of $0.94 \mu \mathrm{g}$ $\left(4.8 \mu \mathrm{g} / \mathrm{cm}^{2}\right)$ PMeT modified electrode in $0.1 \mathrm{M}$ TBAHFP acetonitrile solution and concomitant resonator frequency variation (dotted line, right hand $Y$-axis). Initial and final potential $-0.4 \mathrm{~V}$, vertex potential $1.2 \mathrm{~V}$, scan rate $20 \mathrm{mV} / \mathrm{s}$.

performed stopping the oxidation potential at $1.2 \mathrm{~V}$, that is at potential values where the electropolymerization does not take place. Relevant CV and EQCM plots are reported in Fig. 2a. Note that now the higher amplification of current signals allows a better resolution of the plot with respect to Fig. 1.

A sharp oxidation peak is observed at $0.7 \mathrm{~V}$ in the forward scan to which two reduction peaks are associated in the backward scan. This agrees with the literature [31,37] which describes a two-electron process in one step for the oxidation and two single and separated processes for the reduction. This has been interpreted within the framework of the polaron-bipolaron model, where the bipolaron is formed directly during oxidation, whereas the cathodic process is a two-step reaction involving first the reduction of the bipolaron to polaron and then the return to neutral PMeT [37] according to the following scheme:

$$
\begin{aligned}
& \mathrm{PMeT}^{(\mathrm{a})} \rightarrow \mathrm{PMeT}^{2+}+2 \mathrm{e}^{-} \text {(ox.) } \\
& \mathrm{PMeT}^{2+}+\mathrm{e}^{-} \rightarrow \mathrm{PMeT}^{+} \text {(red.1) } \\
& \mathrm{PMeT}^{+}+\mathrm{e}^{-} \rightarrow \text { PMeT (red.2) }
\end{aligned}
$$

(a) Denotes portion of the polymer chains containing, after oxidation, two positive ionic charges.

The presence of more peaks could also agree with a certain regio-regularity of the polymer as reported recently in the literature, for instance, for the case of some poly-(3alkylthiophenes) [38].
The relevant EQCM pattern shows the frequency changes related to the doping-undoping with the supporting electrolyte anion, according to reaction (1). The simplest model, postulated for analogous systems [39-41], considers the counter ions as the only mobile species. In this approximation, with no solvent transfer, the mass change measured corresponds to $0.028 \mu \mathrm{mol} / \mathrm{cm}^{2} \mathrm{~mol}$ of $\mathrm{BF}_{4}{ }^{-}$which balances roughly the number of positive charges produced by the oxidation process of PMeT and quantified by integration of the oxidation peak. From the amount of polymer deposited, the charge fraction $(\delta)$ for monomer unit is 0.21 . This result agrees with the literature $[28,31,32]$ where $\delta$ ranges between 0.12 and 0.33 , depending on the anion and reaction conditions. Fig. $2 b$ shows the $C V$ and EQCM patterns recorded using TBAHFP instead of TBATFB as supporting electrolyte. Mass changes for doping-undoping in the two different supporting electrolytes are listed in Table 1; note that relevant $\delta$ values do not change significantly for the two cases. These data are useful for the proper preparation of the composite with AQ55 in order to evaluate the mass ratio between AQ55 and PMeT so that, when PMeT is oxidized its positive charges should neutralize the negative charges of the sulphonic groups of AQ55.

\subsection{Mixed AQ55/PMeT films}

The mixed film was prepared on Pt-quartz crystal electrode by potentiostatic electrodeposition at $1.8 \mathrm{~V}$ of MeT on

Table 1

Frequency and mass changes, mass deposited and charge fraction values $(\delta)$ for PMeT, obtained by using two different supporting electrolyte anions in acetonitrile solutions

\begin{tabular}{llllll}
\hline $\begin{array}{l}\text { Supporting electrolyte } \\
\text { counter-ion }\end{array}$ & $\begin{array}{l}\text { Molar mass }(\mathrm{g} / \mathrm{mol}, \\
\text { counter-ion) }\end{array}$ & $\begin{array}{l}\Delta f(\mathrm{~Hz})^{\mathrm{a}} \text { measured at } \\
\text { the doped state }\end{array}$ & $\begin{array}{l}\Delta m_{\text {(exp) }}(\mathrm{ng})^{\mathrm{b}} \text { corresponding } \\
\text { experimental mass }\end{array}$ & $\begin{array}{l}\text { PMeT }(\mu \mathrm{g}) \text { mass } \\
\text { deposited }\end{array}$ & $\begin{array}{l}\delta \text { charges/monomer } \\
\text { unit }\end{array}$ \\
\hline $\mathrm{BF}_{4}{ }^{-}$ & 87 & 446 & $487\left(5.60 \times 10^{-9}\right)$ & 2.64 & 0.21 \\
$\mathrm{PF}_{6}{ }^{-}$ & 145 & 213 & $232\left(1.60 \times 10^{-9}\right)$ & 0.94 & 0.17 \\
\hline
\end{tabular}

${ }^{a}$ Frequency change measured when changing the polymer from the reduced to the oxidized state (doping/undoping).

b Calculated from column 3, by applying the Sauerbrey equation; in parenthesis mol of counter ion. Each value is related to a different quantity of PMeT (see next column). 


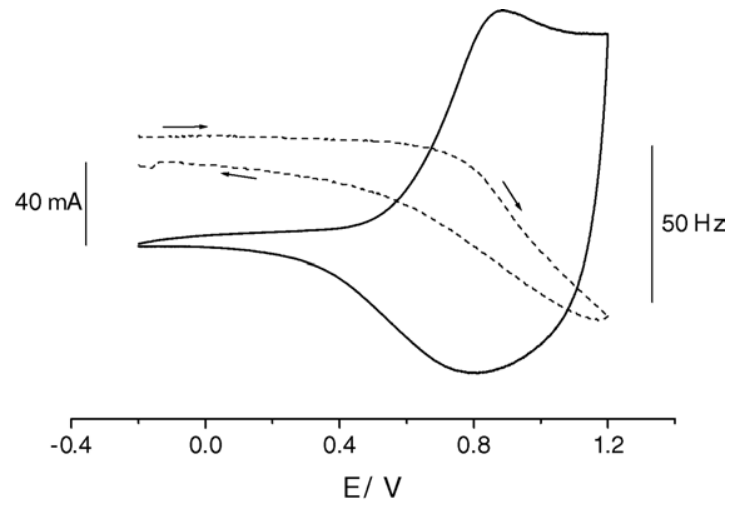

Fig. 3. Cyclic voltammogram (full line) obtained for a PMeT/AQ55 coated Pt-quartz crystal electrode in $0.1 \mathrm{M}$ TBATFB acetonitrile solution and concomitant frequency variation (dotted line which refers to right $Y$-axis). Initial and final potential $-0.2 \mathrm{~V}$, vertex potential $1.2 \mathrm{~V}$, scan rate $20 \mathrm{mV} / \mathrm{s}$.

an electrode pre-coated with a thin film of AQ55 (see Section 2). Such a potential is about $50 \mathrm{mV}$ less positive than the potential of the oxidation peak of monomeric MeT [32]. The risk of overoxidation of PMeT at this potential should be negligible in our experimental conditions, since we operate in accurately dried acetonitrile; in fact, the addition of water or other nucleophiles is required to cause overoxidation at such a potential value [42]. The amount of PMeT deposited was measured and controlled by EQCM frequency changes and coulometric integration of the current. Such a strict control of the deposition process is indeed much more difficult and less accurate if the deposition is performed by cyclic voltammetry. Usually, the process was stopped when the frequency decrease was $3.5 \mathrm{kHz}$. Such a value was calculated on the basis of the monomer mass and charge fraction $(\delta=0.21)$, in order to introduce into the composite an amount of positive charges approximately equal to the number of negative charges of the $\mathrm{SO}_{3}{ }^{-}$groups initially present in the AQ film.

Fig. 3 shows the $\mathrm{CV}$ and EQCM patterns recorded after transferring into $0.1 \mathrm{M}$ TBATFB acetonitrile solution a $\mathrm{Pt}$-quartz electrode coated with the AQ55/PMeT mixed film. The CV pattern is characterized by an oxidation-reduction peak system similar to the one obtained for PMeT alone; however, the oxidation peak is now shifted towards more positive potential values $\left(E_{\mathrm{pa}}=0.870 \mathrm{~V}\right.$ compared with $0.700 \mathrm{~V}$ for $\mathrm{PMeT}$ alone). In the reduction, one broad reduction peak is observed. This cyclic voltammetric behavior indicates that PMeT keeps its electroactivity also in the composite, keeping the ability to switch from conductive to insulating after reduction, as put in evidence by the high charging current observed at a switching potential of $1.2 \mathrm{~V}$. These characteristics in comparison with $\mathrm{CV}$ patterns reported in the literature for overoxidized PMeT [42] exclude the eventuality of an overoxidation of the conducting polymer under the experimental conditions used in the present work.

In principle, the positive charges produced by the oxidation of PMeT in the composite could be neutralized by one

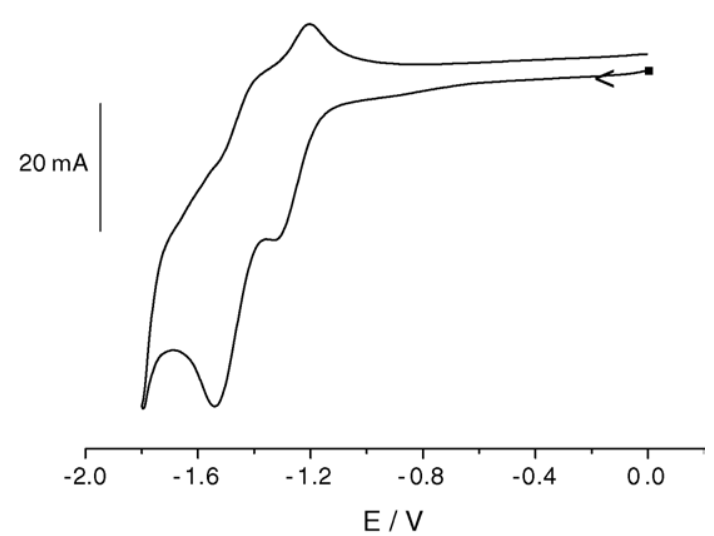

Fig. 4. Cyclic voltammogram obtained for a PMeT/AQ55 modified GCE after 30 min equilibration in $10^{-4} \mathrm{MOs}(\mathrm{bpy})_{3}{ }^{2+}, 0.1 \mathrm{M}$ TBATFB acetonitrile solution. Initial and final potential $0.0 \mathrm{~V}$, vertex potential $-1.8 \mathrm{~V}$, scan rate $50 \mathrm{mV} / \mathrm{s}$.

(or both) of the following processes:

$$
\begin{aligned}
& {\left[\left(\mathrm{AQ}_{-} \mathrm{SO}_{3}{ }^{-} \mathrm{TBA}^{+}\right)(\mathrm{PMeT})\right]+\mathrm{BF}_{4}{ }^{-}} \\
& \quad \leftrightarrows\left[\mathrm{AQ}-\mathrm{SO}_{3}{ }^{-} \mathrm{PMeT}^{+}\right]+\mathrm{TBA}^{+} \mathrm{BF}_{4}{ }^{-}+\mathrm{e}^{-} \\
& {\left[\left({\mathrm{AQ}-\mathrm{SO}_{3}}^{-} \mathrm{TBA}^{+}\right)(\mathrm{PMeT})\right]+\mathrm{BF}_{4}^{-}} \\
& \quad \leftrightarrows\left[\left(\mathrm{AQ}^{-} \mathrm{SO}_{3}{ }^{-} \mathrm{TBA}^{+}\right)\left(\mathrm{PMeT}^{+} \mathrm{BF}_{4}^{-}\right)\right]+\mathrm{e}^{-}
\end{aligned}
$$

EQCM data shown in Fig. 3 indicate that, in the composite, the oxidation of the polymer reflects in a decrease of frequency, that is an increase of mass; this supports the idea that, under these experimental conditions, reaction (6) is the prevailing process. At variance, for instance, with the composite Nafion-poly(aniline) in water solutions [12] for which the permselectivity of Nafion determines anion exclusion for the AQ55-PMeT case the entrance of the $\mathrm{BF}_{4}{ }^{-}$anion is, at least partially, required to neutralize the positive charges generated by PMeT oxidation. The positive shift of the oxidation peak potential in the AQ55-PMeT film with respect to PMeT alone agrees with the observation that, in the composite, the ionic repulsion by the sulphonic groups hinders anyway the entrance of the supporting electrolyte anion and makes the oxidation more energy demanding. Experiments performed with GC disks electrodes indicate that the $\mathrm{CV}$ of the composite deposited both galvanostatically and potentiostatically on a GC electrode pre-coated with AQ55 are fully comparable with those obtained at AQ55 pre-coated Pt-quartz electrodes.

\subsection{Incorporation of electroactive cations}

Fig. 4 shows the voltammetric pattern recorded from $0.0 \mathrm{~V}$ to $-1.8 \mathrm{~V}$ at a GCE coated with the AQ55/PMeT film after dipping in $\left.10^{-4} \mathrm{M} \mathrm{Os(bpy)}\right)_{3}{ }^{2+}$ solution. Peak currents increase with dipping time up to reaching, after about $30 \mathrm{~min}$, the voltammetric pattern shown in Fig. 4. This indicates the 
uptake of the cationic complex by the reduced composite film according to the following reaction:

$$
\begin{aligned}
& 2\left[\left(\mathrm{AQ}^{-\mathrm{SO}_{3}}{ }^{-} \mathrm{TBA}^{+}\right)(\mathrm{PMeT})\right]+\mathrm{Os}(\mathrm{bpy})_{3}{ }^{2+} \\
& \quad \leftrightarrows\left\{\left[\left(\mathrm{AQ}-\mathrm{SO}_{3}{ }^{-}\right)_{2} \mathrm{Os}(\mathrm{bpy})_{3}{ }^{2+}\right] \mathrm{PMeT}\right\}+2 \mathrm{TBA}^{+}
\end{aligned}
$$

Note that, in the case of the AQ55-PMeT film, the incorporation of the cation takes place at potential values close to $0 \mathrm{~V}$ versus $\mathrm{Ag} / \mathrm{AgCl}(\mathrm{KCl}$ sat $)$, that is a potential value much more accessible than the very negative value of $-2.2 \mathrm{~V}$ required for introducing negative charges (and therefore cation-exchange properties) in films of PMeT alone [43]. In the potential window explored in Fig. 4, the conducting polymer is, in fact, always in its reduced (neutral) state.

The observed ion-exchange incorporation agrees with the uptake of the Os(bpy) ${ }_{3}{ }^{2+}$ complex, described in the literature for GC electrodes coated with AQ55 alone [26]; the two reduction peaks in Fig. 4 correspond, in fact, to the stepwise reduction of incorporated $\mathrm{Os}(\mathrm{bpy})_{3}{ }^{2+}$ to $\mathrm{Os}(\mathrm{bpy})_{3}{ }^{+}$and Os(bpy $)_{3}{ }^{\circ}$ [26], with $E_{1 / 2}=\left(E_{\mathrm{pa}}+E_{\mathrm{pc}}\right) / 2$ equal to -1.250 and $-1.450 \mathrm{~V}$, respectively.

Curve (a) in Fig. 5 shows that a similar pattern is obtained soon after the transfer of the AQ55/PMeT coated GC (loaded in $10^{-4} \mathrm{M} \mathrm{Os}(\mathrm{bpy})_{3}{ }^{2+}$ ) into $0.1 \mathrm{M}$ TBATFB acetonitrile electrolyte with no Os(bpy $3_{3}{ }^{2+}$ added to the electrolyte solution.

As shown by curve (b) in Fig. 5 (which is zoom in of the negative going branch of the CV in the inset of Fig. 5), the CV pattern changes significantly after the electrochemical oxidation of the PMeT moiety of the composite. Comparison with curve (a) shows in fact, that the oxidation of the composite at $1.5 \mathrm{~V}$ causes a decrease of the Os(bpy $)_{3}{ }^{2+}$ signals. Following scans in the $+1.5 \mathrm{~V}$ to $-1.8 \mathrm{~V}$ region gave, as final result, curve (b) in Fig. 5, which is finally stable with time.

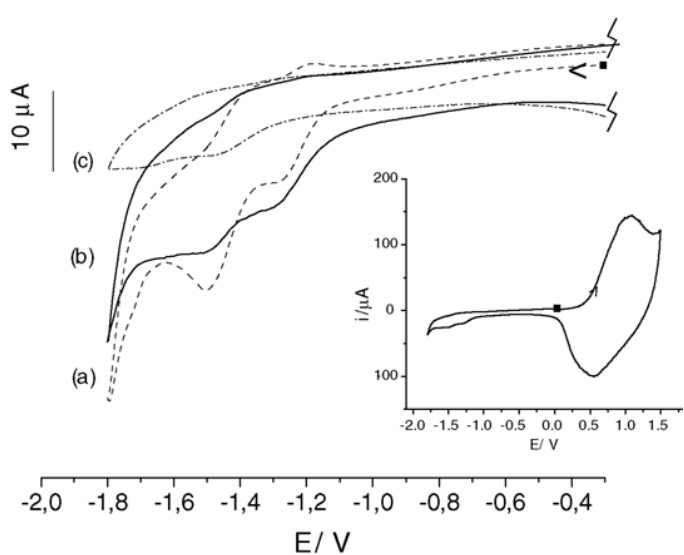

Fig. 5. Cyclic voltammograms of the modified GCE of Fig. 4 after transfer to acetonitrile solutions, with no $\mathrm{Os}(\mathrm{bpy})_{3}{ }^{2+}$ dissolved, scan rate $50 \mathrm{mV} / \mathrm{s}$; (a) starting potential $0.0 \mathrm{~V}$, vertex potential $-1.8 \mathrm{~V}$, final potential $0.0 \mathrm{~V}, 0.1 \mathrm{M}$ TBATFB acetonitrile solution; (b) zoom of the CV in the inset, starting potential $0.0 \mathrm{~V}$, positive vertex potential $+1.5 \mathrm{~V}$, negative vertex potential $-1.8 \mathrm{~V}$, final potential $0.0 \mathrm{~V}, 0.1 \mathrm{M}$ TBATFB acetonitrile solution; (c) as (b), but in acetonitrile with no TBATFB added. Inset: complete picture of the full line cyclic voltammogram.

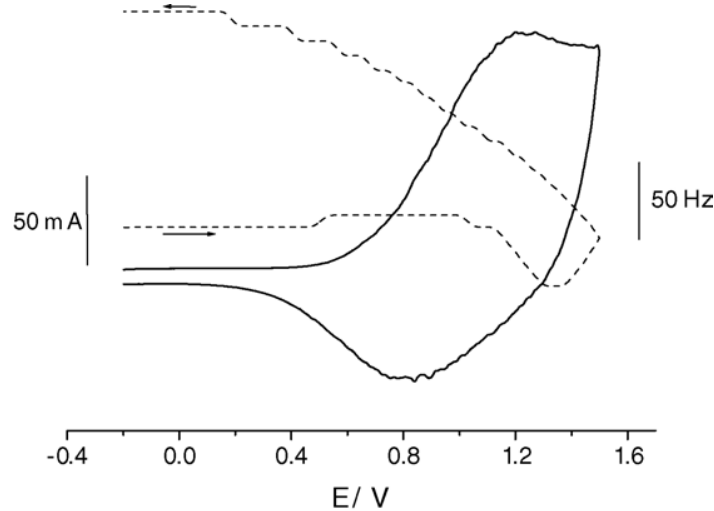

Fig. 6. Cyclic voltammogram (full line, left hand $Y$-axis) and concomitant resonator frequency variations (dashed line, right hand $Y$-axis) of the PMeT/AQ55 coated Pt-quartz crystal electrode loaded with Os(bpy) ${ }_{3}{ }^{2+}$ $10^{-4} \mathrm{M}$, and transferred in $0.1 \mathrm{M}$ TBATFB acetonitrile solution. Initial and final potential $-0.2 \mathrm{~V}$, vertex potential $1.5 \mathrm{~V}$, scan rate $20 \mathrm{mV} / \mathrm{s}$.

This indicates a partial release of the osmium complex since the Os(bpy $)_{3}{ }^{2+}$ reduction signal is lower, but still detectable even after PMeT oxidation. These evidences suggest that the positive charges generated by PMeT oxidation are neutralized partly by the release of some Os(bpy $)_{3}{ }^{2+}$ and partly by the uptake of some supporting electrolyte anion. This is summarized by the following simplified scheme:

$$
\begin{aligned}
& \left\{\left[\left(\mathrm{AQ}-\mathrm{SO}_{3}{ }^{-}\right)_{2} \mathrm{Os}(\mathrm{bpy})_{3}{ }^{2+}\right] \mathrm{PMeT}\right\} \\
& \quad \leftrightarrows\left[\left(\mathrm{AQ}-\mathrm{SO}_{3}{ }^{-}\right)_{2}(\mathrm{PMeT})^{2+}\right]^{(\mathrm{a})}+\mathrm{Os}(\mathrm{bpy})_{3}{ }^{2+}+2 \mathrm{e}
\end{aligned}
$$

$$
\begin{aligned}
& \left\{\left[\left(\mathrm{AQ}^{-} \mathrm{SO}_{3}{ }^{-}\right)_{2} \mathrm{Os}(\mathrm{bpy})_{3}{ }^{2+}\right] \mathrm{PMeT}\right\}+\mathrm{BF}_{4}{ }^{-} \\
& \quad \leftrightarrows\left\{\left[\left(\mathrm{AQ}-\mathrm{SO}_{3}{ }^{-}\right)_{2} \mathrm{Os}(\mathrm{bpy})_{3}{ }^{2+}\right]\left(\mathrm{PMeT}^{+} \mathrm{BF}_{4}{ }^{-}\right)\right]+\mathrm{e}
\end{aligned}
$$

(a) Denotes portion of the polymer chains containing, after oxidation, two positive charges.

Curve (c) in Fig. 5 shows the zooming in the CV recorded as in the inset, but after transfer in pure acetonitrile without added supporting electrolyte (containing no Os(bpy) $3^{2+}$ in solution). Note that even dry-distilled acetonitrile can contain traces of water which can partly act as supporting electrolyte. Under these conditions, the absence of added electrolyte reflects in a further and more effective release of $\mathrm{Os}(\mathrm{bpy}) 3^{2+}$, even if the CV signal of the osmium complex does not disappear completely.

The EQCM curve shown in Fig. 6 focuses on frequency changes associated with the oxidation of the composite loaded in $\mathrm{Os}(\mathrm{bpy})_{3}{ }^{2+}$ solution and transferred into $0.1 \mathrm{M}$ TBATFB acetonitrile solution. This result confirms that an overall decrease in the mass is associated with the oxidation. At the beginning of the oxidation, the quartz resonator detects a slight decrease in frequency (increase in mass) followed by a more evident overall increase in frequency (decrease in mass) which becomes higher when proceeding further with the composite oxidation. 


\subsection{SEM investigations on the morphology of the film}

In order to further investigate the possible reasons behind the only partial release of incorporated redox cations, the morphology of the mixed film was examined by SEM analyses. Fig. 7A-C compares SEM images obtained on cross sec-
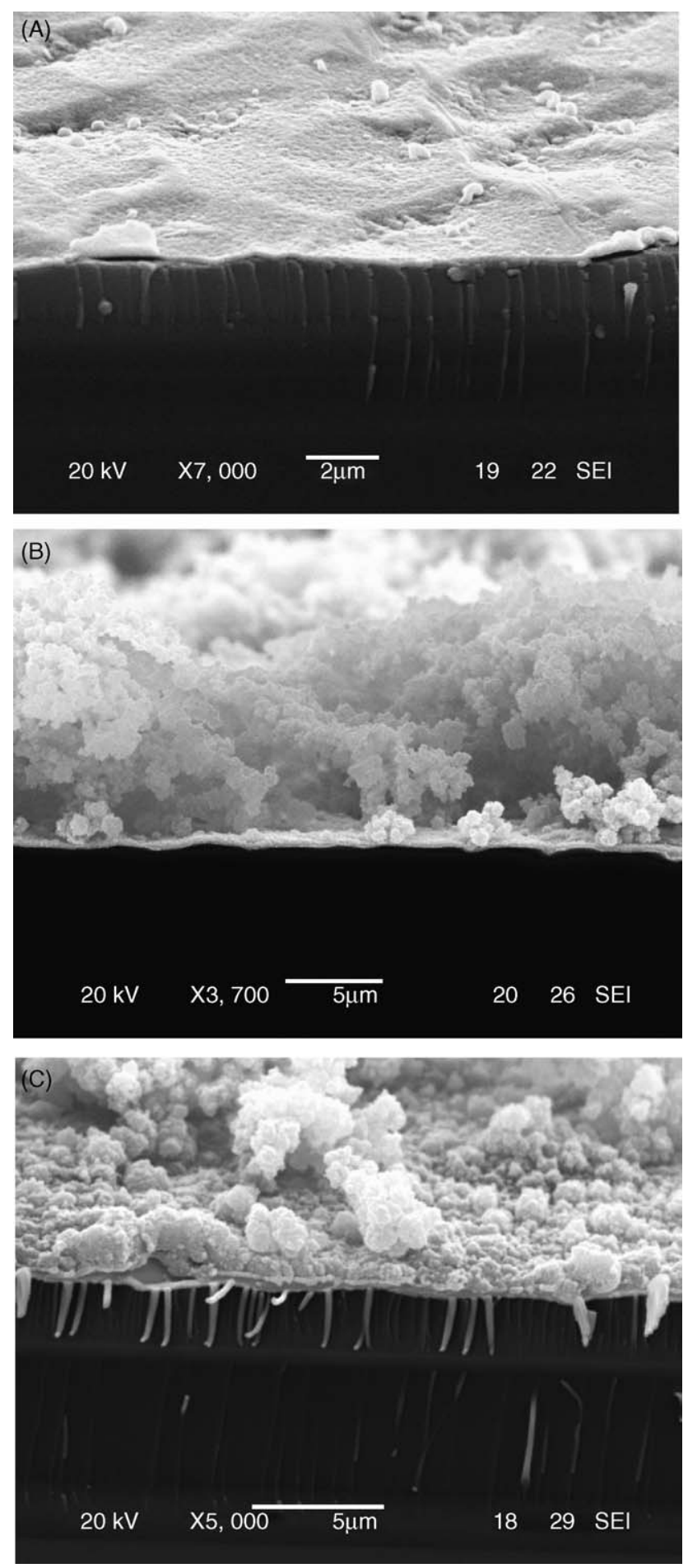

Fig. 7. SEM images of cross sections of films deposited on a Pt-coated quartz crystal: (A) AQ55; (B) PMeT; (C) AQ55/PMeT. tions of AQ55 and PMeT films deposited alone (Fig. 7A and $\mathrm{B}$, respectively) or as composite (Fig. 7C). All the pictures show cross sections of films deposited on Pt electrodes on quartz and fractured at room temperature. Fig. 7A indicates that the AQ55 coating forms a rather compact and homogeneous film, with thickness corresponding approximately to the value expected on the basis of the amount deposited and polymer density [24]. Such a compact morphology agrees with the good ion-exchange permselectivity and low swelling degree which characterize this coating when used in acetonitrile solutions [24-26]. The fracturing of the film at room temperature causes some polymer "beards" to extend in the fractured area; fracturing of the material at low temperature, after dipping in liquid nitrogen, lowers this effect (picture not shown).

On the other hand, films of PMeT alone (Fig. 7B) display a sponge-like structure made of thick aggregates of small particles extended over a rather large thickness. This agrees with the morphology shown by Waltman et al. [32] for top SEM view of PMeT coatings.

Fig. 7C shows the cross section of the AQ55/PMeT mixed film. The image indicates that the composite is really made of a bi-layer where the AQ55 thin layer is in direct contact with the support material and PMeT is deposited on the outer face of the poly-(ester-sulphonated) film. In such a bi-layer, the two polymeric components keep their individual characteristics almost unaltered: thin and compact the AQ55 layer, grainy and thick the PMeT one. These observations explain the ion-exchange behavior of the mixed film. When the PMeT layer is oxidized, $\left[\mathrm{Os}(\mathrm{bpy})_{3}\right]^{2+}$ is only partially released and the majority of the cationic complex remains entrapped in the AQ55 layer, because of ionic repulsion by the now positively charged $\mathrm{PMeT}^{n+}$ layer.

The fraction of the complex which is released probably corresponds to the fraction of $\left[\mathrm{Os}(\mathrm{bpy})_{3}\right]^{2+}$ which was initially incorporated at the interface where AQ55 and PMeT are in direct contact; this is in fact the part of the polymer bi-layer where self-neutralization between sulphonic groups and the positive charges oxidized PMeT can occur.

As shown before in Fig. 5, the release of the osmium complex in pure acetonitrile is more efficient; this can be explained by taking into account that the oxidation of PMeT to produce the positively charged film is partially hindered by the lack of supporting electrolyte; this can cause a slower rate in the oxidation process and the introduction of a smaller final number of positive charges at the end of the oxidation process.

\section{Conclusions}

The AQ55/PMeT composite is stable in acetonitrile solutions, displaying ion-exchange properties which can be modulated electrochemically, but only in the portion of the AQ55/PMeT mixture where an intimate contact between ionomer and conductive polymer is possible. This fraction 
increases when operating in the absence of supporting electrolyte added to the acetonitrile solution.

Really, uptake and entrapment of redox cations are ruled mainly by the oxidation state of the PMeT layer: when neutral, it is permeable to cations (loading), while when oxidized, it partially entraps cations within the bi-layer.

The results of the present work indicate that, in order to achieve the quantitative control of the release of incorporated cations in mixed films, it is important to achieve a contact between ionomer and conducting polymer as intimate as possible. This could be obtained by preparing ultrathin bi-layers, by resorting, for instance, to Langmuir-Blodgett techniques which can be employed for preparing ionomer permselective films as thin as few nanometers [44,45]. Further studies on such a possibility are presently in progress.

\section{Acknowledgements}

This work was supported by MIUR(Rome). We thank Eastman Italia for the AQ55 sample. The authors wish to thank an anonymous reviewer whose suggestions were helpful for gaining insight in the present work.

\section{References}

[1] P. Ugo, in: C. Grimes (Ed.) Encyclopedia of Sensors, American Scientific Publisher, in press.

[2] M.W. Espenscheid, C.R. Martin, J. Electroanal. Chem. 189 (1985) 73.

[3] M.W. Espenscheid, C.R. Martin, Electroanalysis 1 (1989) 93.

[4] P. Ugo, F.C. Anson, Anal. Chem. 61 (1989) 1799.

[5] R. Zumbrunnen, F.C. Anson, J. Electroanal. Chem. 152 (1983) 111.

[6] J. Bobacka, Z. Gao, A. Ivaska, A. Lewenstam, J. Electroanal. Chem. 368 (1994) 33.

[7] A. Lewenstam, J. Bobacka, A. Ivaska, J. Electroanal. Chem. 368 (1994) 23.

[8] F. Fan, A.J. Bard, J. Electrochem. Soc. 133 (1986) 301.

[9] T.P. Gbatu, O. Ceylan, K.L. Sutton, J.F. Rubinson, A. Galal, J.A. Caruso, H.B. Mark, Anal. Commun. 36 (1999) 203.

[10] T. Hirai, S. Kuwabata, H. Yonegani, J. Electrochem. Soc. 135 (1988) 1132.

[11] G. Nagasubramanian, S.D. Stefano, J. Moacanin, J. Phys. Chem. 90 (1986) 4447.
[12] D. Orata, D.A. Buttry, J. Electroanal. Chem. 257 (1988) 71.

[13] R.M. Penner, C.R. Martin, J. Electrochem. Soc. 133 (1986) 310.

[14] T. Shimidzu, A. Ohtani, T. Iyoda, K. Honda, J. Electroanal. Chem. 224 (1987) 123.

[15] J. Wu, X. Yu, H. Lord, J. Pawliszyn, Analyst 125 (2000) 391.

[16] Q.X. Zhou, L.L. Miller, J.R. Valentine, J. Electroanal. Chem. 261 (1989) 147

[17] J. Wang, Z. Sun, Z. Lu, J. Electroanal. Chem. 310 (1991) 269.

[18] G. Liljegren, J. Petterson, K.E. Markides, L. Nyholm, Analyst 127 (2002) 591

[19] J. Wu, J. Pawliszyn, J. Chromatogr. A 909 (2001) 37.

[20] R.S. Deinhammer, M.D. Porter, K. Shimazu, J. Electroanal. Chem. 387 (1995) 35.

[21] R.S. Deinhammer, K. Shimazu, M.D. Porter, Anal. Chem. 67 (1995) 237.

[22] T. Nagaoka, M. Fujimoto, Y. Uccida, K. Ogura, J. Electroanal. Chem. 336 (1992) 45.

[23] P. Nikitas, J. Electroanal. Chem. 484 (2000) 137.

[24] B. Brunetti, P. Ugo, J. Electroanal. Chem. 460 (1999) 38.

[25] T. Gennett, W.C. Purdy, Anal. Chem. 62 (1990) 2155.

[26] J. Hanzlik, P. Ugo, S. Daniele, G.A. Mazzocchin, J. Electroanal. Chem. 404 (1996) 89.

[27] F. Nguyen, F.C. Anson, Electrochim. Acta 44 (1998) 239

[28] A. Galal, Electroanalysis 10 (1998) 121.

[29] R.B. Kaner, in: R.G. Linford (Ed.), Electrochemical Science and Technology of Polymers, vol. 2, Elsevier Applied Science, London, 1990, p. 97.

[30] S. Servagent, E. Vieil, J. Electroanal. Chem. 280 (1990) 227.

[31] C. Visy, J. Lukkari, J. Kankare, J. Electroanal. Chem. 319 (1991) 85.

[32] R.J. Waltman, J. Bargon, A.F. Diaz, J. Phys. Chem. 87 (1983) 1459.

[33] F.H. Burstall, F.P. Dwyer, E.C. Gyrafs, J. Chem. Soc. (1950) 953.

[34] S. Bruckenstein, M. Shay, Electrochim. Acta 30 (1989) 1295.

[35] D. Buttry, in: A.J. Bard (Ed.), Electroanalytical Chemistry, vol. 17, Marcel Dekker, New York, 1991, p. 1.

[36] P. Ugo, L.M. Moretto, S. Bellomi, V.P. Menon, C.R. Martin, Anal. Chem. 68 (1996) 4160.

[37] Z.G. Xu, G. Horowitz, J. Electroanal. Chem. 335 (1992) 123.

[38] M. Skompska, A. Szkurlat, Electrochim. Acta 46 (2001) 4007.

[39] A.R. Hillman, M.J. Swann, S. Bruckenstein, J. Electroanal. Chem. 291 (1990) 147.

[40] J.R. Reynolds, N.S. Sundaresan, M. Pomerantz, S. Busak, C.K. Baker, J. Electroanal. Chem. 251 (1988) 355.

[41] P.T. Varineau, D.A. Buttry, J. Phys. Chem. 91 (1987) 1292.

[42] U. Barsch, F. Beck, Electrochim. Acta 41 (1996) 1761.

[43] A.-N. Chowdhury, Y. Harima, Y. Kunugi, K. Yamashita, Electrochim. Acta 41 (1996) 1993.

[44] P. Ugo, L.M. Moretto, F. Vezzà, Chem. Phys. Chem. 3 (2002) 917.

[45] P. Ugo, P. Bertoncello, F. Vezzà, Electrochim. Acta 49 (2004) 3785. 